Ophthalmologica 1939;96:377-379

\title{
Register rerum vol. 96
}

Die fett gedruckten Zahlen bedeuten Originalarbeiten. - Les chiffres gras indiquent des travaux originaux. - Black ciphers signify original papers. Bb. $=$ Buchbesprechungen - Book Notices - Livres nouveaux.

A

Ablatio retinae 125, 126, 129

ser $\aleph^{1 / 8 e}$ - bei orbitaler Entzün-dung 301

Azetylcholinesterase-Gehalt d. sub-retinalen Flüssigkeit bei - 348

Heilung einer - trotz Aphakie und Nystagmus 307

Altindische Ophthalmologie 189

Aneurin 219

Arachnodaktylie, Linsenektropie bei

- 174 Arachnoitis optico-chiasmatica 67 - traumatischen Ursprungs 336 Atcus lipoides bei Buphthalmus 192 Äther bei Herpes corneae 189 Augenfärbung 61 Augenhintergrund, Fundus albipunc-

tatus cum Hemeralopia 110 Azetylcholinesterase-Gehalt deГ sub-

retinalen Flüssigkeit bei ruptu-

rellen Netzhautablösungen 348

B

Biochemie des Auges 37 (Bericht) Blepharochalasis, Operation de $Г-295$ Blepharoplastik 294 Blepharorrhaphie 293 Blepharospasmen, ticartige 66 Blutdruckbestimmungen in den Netzhautgefäßen 15, 124, 315 Blutzyste deГ Orbita 307 Buphthalmus, Arcus lipoides bei -192

C

Chemosís conjunctivae, partielle 298

Chorioidealtuberkel 65

Color Fatigue in the Peripheral Visual Field 137, 267

Conjunctiva, bakteriologische Befunde auf der - 128

Cornea, Biochemie der - 58

- Durchblutung der - 122

- Gefäßneubildung in der - bei Keratitis tuberculosa 301

eine pigmentierte Hornhautlinie bei Pterygium 112

Verfärbung der - durch ein Adrenalinpräparat 306

Degeneration der - 306

Cornea, erbliche Dystrophien der - 112, 199 (Bb.)

punkt- und ringförmige Entartung der - 34

Vitamin-A-Therapie bei Erkran-kungen der - 3

$\mathrm{D}$

Degeneratio hyaloideo-retinális Ill Diabetes, Rückbildung von Linsen-

trübung bei - 174 Diathermiestichelung des Corpus ci- 
liare bei Glaukom 117 Dinitrophenolstar 170

E Eisensplitterverletzung 130 Entropionoperation 292 Epitheleinsprossung in die Vorderkammer 29, 302 Erholungszeit nach Einwirkung far-

biger Reize 137, 267 Exophthalmus, pulsierender 129, 306

F

Farbenermüdung, Erholungszeit nach

- $\quad$ im peripherischen Gesichtsfeld137, 267

Febris uveoparotidea 65 Foster-Kennedys Syndrom 119

G

Gastroretinaler Reflex 355 Gefäßneubildungen in der Hornhaut

bei Keratitis tuberculosa 301 Glaskorper 185 (Bericht)

- $\quad$ Biochemie des -57 Glaucitsalbe, Verfärbung der Cornea

durch - 306 Glaukom 129

- $\quad$ und innere Sekretion 310

Sekundär-Gl. bei epibulbären Tu-moren 312

nach Starextraktion 297

Diathermiestichelung des Corpus ciliare bei -117

Glaukomoperationen, Katarakt nach

- $\quad$ 297Glykolysemechanismus der Linse 38

378

Register rerum

$\mathrm{H}$

Пaftgläser 127

HémoГГ agie expulsive, Verhütung der - duГch äquatorische Trepanation 201

Hemianopsie, Vortäuschung einer ho-monymen - 110

He par in 317

Herpes corneae, Aether bei - 189

Herpes erythematodes, Retinalbefund bei -307

Hyaline Konkretionen in der Papule 298

Hypophorie 303

Hypopyoníritis, rezidivierende 132, 297

I

Innere Sekretion und Glaukom 310 Inosit in den Augengeweben 60 Instruments 375 (Bb.)

Intraokulare Spannung bei Tumoren

315 Iridektomia tangentialis 115 Iriszyste, posttraumatische - in statu

renascendi 298 Iritis und Geschwürsbildung der Vulva

132

$\mathrm{K}$

Kammerwasser, Biochemie des - 55 Karnosin 60 Katarakt 172

ein ungewöhnlicher Fall von dia-betischer - 98

Dinitrophenolstar 170

nach Glaukomiridektomie 297

Vitamin $7 / 8$ bei Altersstar 123 Kataraktoperationen 176, 312

Glaukom nach - 297 
Tumorwachstum nach -312 Keratitis familialis oder herpetifor-

mis 109

- $\quad$ Lackierer-K. 65

Keratitis parenchymatosa anaphylac-

tica 301 Keratitis tuberculosa, Gefäßneubildung

in der Hornhaut bei - 301 Kératocône fruste 77 Keratoplastik 298

- $\quad$ Lappensicherung bei der durch-greifenden optischen - 155

Keratoskopie mittels des Nordensen-

schen Apparates 84 Koagulierende Substanzen bei Augen-

operationen 318 Korepraxie 126 Krokodiltrãnen 355 Kropf, Chirurgie des - 375 (Bb.)

L

Lancasters Stitch 217 Lappensicherung bei der durchgrei-

fenden optischen Keratoplastik

155 Lackiererkeratitis 65 Lepra der Augenlider 283 Lider 277 (Bericht) Lidödeme 128 Linse 167

(Bericht)

Biochemie der -37

Aneurin (Vitamin B) der - 219 Lumbalpunktion, Augenspiegel- -

befunde bei -311

$\mathrm{M}$

Macula lutea, klinische Bedeutung

der - 191 Maladie des porchers, Augensym-

ptome bei der - 159 Meibomsche Drüsen, Karzinom der

- 22 Melanoblastommetastasen 302 Migråne 371 Myiasis 284

$\mathrm{N}$

Nase, Tuberkulose des Auges und der - 195

Natriumdampflampe 122

Nichtrostende Stahlsorten, Toleranz des Auges gegen - 133

Nicolas-Favre, Parinaudsche Conjunctivitis verursacht durch das Virus von - 321

Nordensenscher Apparat 84

Nystagmus, Bahnen des - 376 (Bb.)

Nystagmus retractorius 304

$\mathrm{O}$

Oberlid, Strangfixation des - 304 Oftalmologia dei paesi caldi - 375

(Bb.) Orbítopalpebralzyste 128

$\mathrm{P}$

Pagetsche Krankheit 297

Parinaudsche Conjunctivitis, verursacht durch das Virus von Nico-las-Favre 321

Phakohårese 178

Photog $\tau$ aphie des vorderen Augenab-schnittes mittels des Nordensen-schen Apparates 84

Pigmentation, ve $\Gamma$ schiedene - des linken und rechten Auges 113

Poikilodermíe 127

Polyzythämie, Fundusveränderungen bei -300

Register rerum

379

Pseudogliom bei einem 2jährigen 
Kinde 298 Pseudotumo $\Gamma$ orbitae 303 Pterygium, eine pigmentierte Hornhautlinie bei - 112

- $\quad$ Operation des - 333Ptosisfamilie 304Ptosisoperation 297Pupille, künstliche 126

$\mathrm{R}$

Raupenhaarverletzung 298, 300 Retina, Biochemie der - 46

Blutdruckbestimmungen in den Gefäßen der - 15, 124, 315

Befund der - bei Erythematodes 307

Retinazysten 309 Retinochorioiditis, akute 310

S Sarkoid Boeck der Augenlider 283 Schielen 130 Schieloperationen, Nachbehandlung nach - 66 Schweinehirtenkrankheít, Augensym-

ptome bei der - 159 Spaltlampenmikroskopie 90, 225 Spermidin in den Augengeweben 60 Spermin in den Augengeweben 60 Stauungspapille 193 Stereoskopische Uebungen 72 (Bb.)

Strahlenbehandlung 129 Syphilis der Augenlider 282

$\mathrm{T}$

Trånenflüssigkeit, Biochemie der -

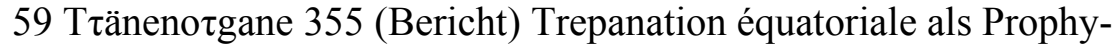

laxe der Hämorrhagie 201 Trichiasis, Operation bei 294 Tuberkulinbehandlung 314 Tuberkulose, Augen- und Nasen-T.

195 Tumoren der Augenlider 285 - intraokuläre Spannung bei - 315 Tumorwachstum nach Starextraktion

312

$\mathrm{V}$

Veråtzungen des Auges 138 (Bb.)

Vitamine 130, 310

Vitamin-A-T'herapie bei Hornhaut-

erkrankungen 3 Vitamin-B2 bei Altersstar 123 Vorderkammer, Epitheleinsprossung

in die - 29, 302 Vulva, Iritis und Geschwürsbildung

der -132

$\mathrm{W}$

Wetter und Patient 72 (Bb.), 112

Z. Z

Zitronensäure im Auge 60, 135 (Bb.) 